\title{
Solusi Persamaan Diferensial Fraksional Riccati dengan Ruas Kanan Konstanta menggunakan Elzaki Decomposition Method dan Analisis Kekonvergenannya
}

\author{
Adrizal Boby Rachman, Eddy Djauhari, M Deni Johansyah \\ Departemen Matematika, Fakultas MIPA, Universitas Padjadjaran, \\ Jl. Raya Bandung Sumedang KM 21 Jatinangor Sumedang 45363 \\ Email: adrzboby@gmail.com, eddy.djauhari@unpad.ac.id, \\ muhamad.deni@unpad.ac.id
}

\begin{abstract}
Abstrak
Persamaan diferensial pada umumnya memiliki orde bilangan asli, namun persamaan diferensial dapat dikembangkan lagi menjadi bentuk orde pecahan. Persamaan diferensial yang memiliki orde bilangan pecahan disebut persamaan diferensial fraksional. Pada penelitian ini penulis mencari solusi persamaan diferensial fraksional non-linear, khususnya persamaan diferensial fraksional Riccati menggunakan Elzaki Decomposition Method (EDM). Elzaki Decomposition Method adalah sebuah metode yang diusulkan oleh Elzaki untuk menyelesaikan persamaan diferensial fraksional non-linear. Metode ini diusulkan berdasarkan modifikasi sederhana dari Adomian Decomposition Method di mana dikombinasikan dengan sebuah transformasi. Selanjutnya dapat dilihat bahwa solusi persamaan diferensial fraksional Riccati berorde $\left(\alpha_{n}\right)$ menghasilkan pola grafik yang mendekati solusi persamaan diferensial fraksional berorde $\left(\alpha_{n}\right)$, sehingga barisan fungsi solusi persamaan diferensial fraksional Riccati konvergen ke fungsi solusi persamaan diferensial fraksional Riccati.

Kata kunci: Persamaan Diferensial Fraksional, Persamaan Diferensial Fraksional Riccati, Elzaki Decomposition Method, Konvergen.
\end{abstract}

\begin{abstract}
Differential equations generally have order of natural numbers, but differential equations can be developed again into the form of fraction order. Differential equations that have a fraction order are called fractional differential equations. In this study, the writer will look for solutions to non-linear fractional differential equations, specifically Riccati fractional differential equations using the Elzaki Decomposition Method (EDM). Elzaki Decomposition Method is a method proposed by Elzaki to solve non-linear fractional differential equations. This method is proposed based on a simple modification of the Adomian Decomposition Method which is combined with a transformation. Furthermore, it can be seen that the solution of the Riccati fractional differential equation produces a graphic pattern that approaches the solution of the fractional differential equation, so that the rows of the Riccati fractional differential equation solution converge to the Riccati fractional differential equation solution.
\end{abstract}

Keywords: Fractional Differential Equation, Riccati Fractional Differential Equation, Elzaki Decomposition Method, Convergent.

2000 Mathematics Subject Classification: 34A08

Submitted: 2020-07-21; Revision: 2020-10-18; Accepted : 2020-10-30 


\section{Pendahuluan}

Seiring dengan berjalannya waktu, ilmu pengetahuan dan teknologi selalu mengalami perkembangan. Di mana disaat terjadi perkembangan tersebut, tak jarang memunculkan beberapa permasalahan yang harus diselesaikan. Ilmu pengetahuan memiliki peran penting, baik itu dalam perkembangan teknologi maupun dalam menyelesaikan permasalahan yang dimunculkan oleh perkembangan teknologi itu sendiri. Salah satu contoh dari ilmu pengetahuan tersebut ialah matematika. Banyak bidang ilmu yang mengacu pada model matematika untuk memecahkan berbagai permasalahan. Persamaan diferensial menjadi salah satu model matematika yang banyak digunakan untuk memecahkan berbagai permasalahan.

Persamaan diferensial adalah suatu persamaan yang memuat variabel bebas, variabel tak bebas, derivatif-derivatif biasa atau diferensial biasa dari variabel tak bebas terhadap variabel bebas-n. Persamaan diferensial pertama kali muncul dengan penemuan kalkulus oleh Newton dan Leibniz. Ditinjau dari peubah bebasnya, persamaan diferensial dapat dibedakan menjadi dua macam, yaitu persamaan diferensial biasa dan parsial, sedangkan persamaan diferensial dilihat dari bentuk fungsi atau pangkatnya juga dibedakan menjadi dua, yaitu persamaan diferensial linear dan persamaan diferensial non-linear.

Persamaan diferensial pada umumnya memiliki orde bilangan asli, namun persamaan diferensial dapat dikembangkan lagi menjadi bentuk orde pecahan. Persamaan diferensial yang memiliki orde bilangan pecahan disebut persamaan diferensial fraksional. Sejarah kalkulus fraksional bermula dari pertanyaan LHpital kepada G.W Leibniz pada tahun 1695 tentang makna dari $\frac{d^{n} y}{d x^{n}}$ untuk n bilangan asli, lalu bagaimana jika $n=\frac{1}{2}$ yang merupakan bilangan fraction/pecahan. Sejak saat itu para Matematikawan terus mengembangkan topik tersebut, seperti L. Euler, P.S Laplace, J.B.J Fourier, N.H. Abel, J. Liouville, B. Riemann, A.K. Grunwald, dan A.V. Letnikov [? ].

Salah satu metode yang dapat digunakan untuk mencari solusi persamaan diferensial fraksional non-linear adalah Elzaki Decomposition Method (EDM) yang merupakan pembaruan dari Metode Dekomposisi. Banyak peneliti yang telah mencari solusi persamaan diferensial fraksional non-linear dengan berbagai metode, seperti menggunakan Adomian Decomposition Method [? ], Metode Dekomposisi [? ], Transformasi Diferensial [? ], Variational Iteration Method [? ] dan Telescoping Decomposition Method [? ]. Dapat dilihat pula Kajian Dasar Integral dan Turunan Fraksional Riemann-Liouvill pada [? ] dan [? ]

Selanjutnya pada penelitian ini penulis mencari solusi persamaan diferensial fraksional non-linear, khususnya persamaan diferensial fraksional Riccati menggunakan Elzaki Decomposition Method (EDM), kemudian dianalisis kekonvergenan barisan fungsi solusi persamaan diferensial fraksional Riccati, serta membuat grafik untuk memperoleh pola dari fungsi solusi persamaan diferensial fraksional Riccati lalu ditampilkan pula grafik hasil pencarian solusi Elzaki Decomposition Method dan grafik solusi eksak.

\section{Metode Penelitian}

Merujuk pada [? ] Persamaan diferensial yang digunakan dalam penelitian ini adalah persamaan diferensial fraksional Riccati dengan bentuk umum sebagai berikut

$$
D^{\alpha} u(t)+A u^{2}(t)+B u(t)=r(t), \quad 0<\alpha \leq 2
$$

di mana A dan B adalah konstanta real.

2.1. Elzaki Decomposition Method. Merujuk pada [? ], Elzaki Decomposition Method adalah sebuah metode yang diusulkan oleh Elzaki untuk menyelesaikan persamaan diferensial fraksional non-linear. Merujuk pada [? ], metode ini diusulkan berdasarkan modifikasi sederhana dari Adomian Decomposition Method di mana dikombinasikan dengan sebuah transformasi yang didefinisikan sebagai berikut

$$
E[f(t)]=v \int_{0}^{\infty} f(t) e^{-\frac{t}{v}} d t=T(v), \quad \forall t>0 .
$$


Teorema 2.1. (Elzaki \& Chamekh, 2018 [? ]) Misalkan T'(v) adalah transformasi (2) dari turunan $f(t)$. Kemudian,

$$
\begin{aligned}
T^{\prime}(v) & =\frac{T(v)}{v}-v f(0) \\
T^{(n)}(v) & =\frac{T(v)}{v^{n}}-\sum_{k=0}^{n-1} v^{2-n+k} f^{(k)}(0), n \geq 1
\end{aligned}
$$

di mana $T^{n}(v)$ adalah transformasi (2) dari turunan ke-n dari fungsi $f(t)$.

Teorema 2.2. (Elzaki \& Chamekh, 2018) Jika $m-1<\alpha m, m \in N$, maka transformasi (2) dari turunan fraksional $D^{\alpha} f(t)$ adalah,

$$
E\left[D^{\alpha} f(t)\right]=T^{\alpha}(v)=\frac{T(v)}{v^{\alpha}}-\sum_{k=0}^{m-1} f^{(k)}(0) v^{2-\alpha+k}
$$

di mana $T(v)$ adalah transformasi (2) dari $f(t)$. Elzaki Decomposition Method mewakili solusi sebagai deret tak hingga

$$
u(t)=\sum_{r=0}^{\infty} u_{r}(t)
$$

dan istilah non-linear $N\left(u(t), u^{\prime}(t)\right)$ terurai menjadi

$$
N\left(u(t), u^{\prime}(t)\right)=\sum_{r=0}^{\infty} A_{r}(t)
$$

di mana

$$
A_{r}(t)=\frac{1}{r !} \frac{d^{r}}{d p^{r}} N\left(\sum_{R=0}^{\infty} P^{r} u_{r}(t), \sum_{R=0}^{\infty} P^{r} u_{r}^{\prime}(t)\right)_{p=0}
$$

adalah polinom Adomian. Iterasi didefinisikan oleh hubungan rekursif

$$
\begin{aligned}
E\left[u_{0}(t)\right] & =\sum_{k=0}^{m-1} u^{(k)} v^{2+k}+v^{\alpha} E[f(t)] \\
E\left[u_{r}(t)\right] & =-v^{\alpha}\left[a_{m} E\left[u_{r-1}^{(m)}(t)\right]+\cdots+a_{1} E\left[u_{r-1}^{\prime}(t)\right]+a_{0} E\left[u_{r-1}(t)\right]\right] \\
& -v^{\alpha} E\left[A_{r-1}(t)\right]
\end{aligned}
$$

\section{Hasil dan Pembahasan}

Diberikan bentuk umum persamaan diferensial fraksional Riccati berorde $\alpha$ berikut

$$
D^{\alpha} u(t)+A u^{2}(t)+B u(t)=k
$$

dengan kondisi awal $u(0)=0$.

Metode pencarian solusi dari persamaan diferensial fraksional berorde $\alpha$ menggunakan Elzaki Decomposition Method yaitu sebagai berikut

$$
D^{\alpha} u(t)=k-A u^{2}(t)-B u(t)
$$

mentransformasi persamaan $(12)$

$$
E\left[D^{\alpha} u(t)\right]=E[k]-A E\left[u^{2}(t)\right]-B E[u(t)]
$$

menyubstitusi persamaan (5) ke persamaan (13)

$$
\begin{aligned}
\frac{T(v)}{v^{\alpha}}-\sum_{k=0}^{m-1} u^{(k)}(0) v^{2-\alpha+k} & =E[k]-A E\left[u^{2}(t)\right]-B E[u(t)] \\
\frac{E[u(t)]}{v^{\alpha}}-\sum_{k=0}^{m-1} u^{(k)}(0) v^{2-\alpha+k} & =E[k]-A E\left[u^{2}(t)\right]-B E[u(t)]
\end{aligned}
$$


menggunakan persamaan (2) untuk menyelesaikan $E[k]$

$$
\begin{aligned}
\frac{E[u(t)]}{v^{\alpha}}-\sum_{k=0}^{m-1} u^{(k)}(0) v^{2-\alpha+k} & =\left[v \int_{0}^{\infty} k e^{-\frac{t}{v}} d t\right]-A E\left[u^{2}(t)\right]-B E[u(t)] \\
\frac{E[u(t)]}{v^{\alpha}}-u^{(0)}(0) v^{2-\alpha+0} & =k v \lim _{a \rightarrow \infty}\left[\int_{0}^{a} e^{-\frac{t}{v}} d t\right]-A E\left[u^{2}(t)\right]-B E[u(t)] \\
\frac{E[u(t)]}{v^{\alpha}}-u(0) v^{2-\alpha} & =k v \lim _{a \rightarrow \infty}\left[\left((-v) e^{-\frac{t}{v}}\right) \mid \begin{array}{c}
a \\
0
\end{array}\right]-A E\left[u^{2}(t)\right]-B E[u(t)] \\
\frac{E[u(t)]}{v^{\alpha}}-u(0) v^{2-\alpha} & =k v \lim _{a \rightarrow \infty}\left[\left((-v) e^{-\frac{a}{v}}\right)-\left((-v) e^{-\frac{0}{v}}\right)\right] \\
& -A E\left[u^{2}(t)\right]-B E[u(t)] \\
\frac{E[u(t)]}{v^{\alpha}}-u(0) v^{2-\alpha} & =k v((0)-(-v))-A E\left[u^{2}(t)\right]-B E[u(t)] \\
\frac{E[u(t)]}{v^{\alpha}}-u(0) v^{2-\alpha} & =k v^{2}-A E\left[u^{2}(t)\right]-B E[u(t)] \\
E[k] & =v \int_{0}^{\infty} k e^{-\frac{t}{v}} d t=k v^{2} .
\end{aligned}
$$

Mengoperasi perkalian kedua ruas dalam persamaan (14) dengan $v^{\alpha}$

$$
\begin{aligned}
v^{\alpha}\left(\frac{E[u(t)]}{v^{\alpha}}-u(0) v^{2-\alpha}\right) & =v^{\alpha}\left(k v^{2}-A E\left[u^{2}(t)\right]-B E[u(t)]\right) \\
E[u(t)]-u(0) v^{2} & =k v^{2+\alpha}-A v^{\alpha} E\left[u^{2}(t)\right]-B v^{\alpha} E[u(t)] \\
E[u(t)] & =(0) v^{2}+k v^{2+\alpha}-A v^{\alpha} E\left[u^{2}(t)\right]-B v^{\alpha} E[u(t)] \\
E[u(t)] & =k v^{2+\alpha}-A v^{\alpha} E\left[u^{2}(t)\right]-B v^{\alpha} E[u(t)] .
\end{aligned}
$$

Berdasarkan persamaan (10) dan (15) diperoleh

$$
\begin{aligned}
E\left[u_{0}(t)\right] & =u^{(0)} v^{2+0}+v^{\alpha} k v^{2} \\
& =k v^{\alpha+2} \\
u_{0}(t) & =\frac{k v^{\alpha+2}}{v^{2}}=k v^{\alpha} \\
& =\frac{k t^{\alpha}}{\Gamma(\alpha+1)} . \\
E\left[u_{i}(t)\right] & =-v^{\alpha}\left[a_{m} E\left[u_{i-1}^{(m)}(t)\right]+\ldots+a_{1} E\left[u_{i-1}^{\prime}(t)\right]+a_{0} E\left[u_{r-1}(t)\right]\right] \\
& v^{\alpha} E\left[A_{r-1}(t)\right], \quad i=1,2,3, \ldots
\end{aligned}
$$

dengan

$$
A_{i}(t)=\frac{1}{i !} \frac{d^{i}}{d p^{i}}\left[u_{0}^{2}+2 p u_{0} u_{1}+p^{2}\left(u_{1}^{2}+2 u_{0} u_{2}\right)+\ldots\right]_{p=0}
$$

cHal ini mengakibatkan

$$
\begin{aligned}
E\left[u_{i}(t)\right] & =-B v^{\alpha} E\left[u_{i-1}(t)\right]-A v^{\alpha} E\left[A_{i-1}(t)\right] \\
E\left[u_{1}(t)\right] & =-B v^{\alpha} E\left[u_{1-1}(t)\right]-A v^{\alpha} E\left[A_{1-1}(t)\right] \\
& =-B v^{\alpha} E\left[u_{0}(t)\right]-A v^{\alpha} E\left[A_{0}(t)\right] \\
& =-B v^{\alpha} E\left[u_{0}(t)\right]-A v^{\alpha} E\left[u_{0}^{2}(t)\right] \\
& =-B v^{\alpha} E\left[\frac{k t^{\alpha}}{\Gamma(\alpha+1)}\right]-A v^{\alpha} E\left[\left(\frac{k t^{\alpha}}{\Gamma(\alpha+1)}\right)^{2}\right] \\
u_{1}(t) & =-k B v^{2 \alpha}-\frac{k^{2} A v^{3 \alpha} \Gamma(2 \alpha+1)}{\Gamma^{2}(\alpha+1)} \\
& =-\frac{k B t^{2 \alpha}}{\Gamma(2 \alpha+1)}-\frac{k^{2} A \Gamma(2 \alpha+1) t^{3 \alpha}}{\Gamma(3 \alpha+1) \Gamma^{2}(\alpha+1)} .
\end{aligned}
$$


Sehingga diperoleh

$$
\begin{aligned}
& E\left[u_{2}(t)\right]=-B v^{\alpha} E\left[u_{2-1}(t)\right]-A v^{\alpha} E\left[A_{2-1}(t)\right] \\
& =-B v^{\alpha} E\left[u_{1}(t)\right]-A v^{\alpha} E\left[A_{1}(t)\right] \\
& =-B v^{\alpha} E\left[-\frac{k B t^{2 \alpha}}{\Gamma(2 \alpha+1)}-\frac{k^{2} A \Gamma(2 \alpha+1) t^{3 \alpha}}{\Gamma(3 \alpha+1) \Gamma^{2}(\alpha+1)}\right]-A v^{\alpha} E\left[2 u_{0}(t) u_{1}(t)\right] \\
& =-B v^{\alpha} E\left[-\frac{k B t^{2 \alpha}}{\Gamma(2 \alpha+1)}-\frac{k^{2} A \Gamma(2 \alpha+1) t^{3 \alpha}}{\Gamma(3 \alpha+1) \Gamma^{2}(\alpha+1)}\right] \\
& -A v^{\alpha} E\left[\frac{2 k t^{\alpha}}{\Gamma(\alpha+1)}\left(-\frac{k B t^{2 \alpha}}{\Gamma(2 \alpha+1)}-\frac{k^{2} A \Gamma(2 \alpha+1) t^{3 \alpha}}{\Gamma(3 \alpha+1) \Gamma^{2}(\alpha+1)}\right)\right] \\
& u_{2}(t)=k B^{2} v^{3 \alpha}+\frac{k^{2} A B v^{4 \alpha} \Gamma(2 \alpha+1)}{\Gamma^{2}(\alpha+1)}+\frac{2 k^{2} A B v^{4 \alpha} \Gamma(3 \alpha+1)}{\Gamma(\alpha+1) \Gamma(2 \alpha+1)} \\
& +\frac{2 k^{3} A^{2} v^{5 \alpha} \Gamma(2 \alpha+1) \Gamma(4 \alpha+1)}{\Gamma(3 \alpha+1) \Gamma^{3}(\alpha+1)} \\
& =\frac{k B^{2} t^{3 \alpha}}{\Gamma(3 \alpha+1)}+\frac{k^{2} A B \Gamma(2 \alpha+1) t^{4 \alpha}}{\Gamma(4 \alpha+1) \Gamma^{2}(\alpha+1)}+\frac{2 k^{2} A B \Gamma(3 \alpha+1) t^{4 \alpha}}{\Gamma(\alpha+1) \Gamma(2 \alpha+1) \Gamma(4 \alpha+1)} \\
& +\frac{2 k^{3} A^{2} \Gamma(2 \alpha+1) \Gamma(4 \alpha+1) t^{5 \alpha}}{\Gamma(3 \alpha+1) \Gamma(5 \alpha+1) \Gamma^{3}(\alpha+1)} \text {. } \\
& E\left[u_{3}(t)\right]=-B v^{\alpha} E\left[u_{3-1}(t)\right]-A v^{\alpha} E\left[A_{3-1}(t)\right] \\
& =-B v^{\alpha} E\left[u_{2}(t)\right]-A v^{\alpha} E\left[A_{2}(t)\right] \\
& =-B v^{\alpha} E\left[u_{2}(t)\right]-A v^{\alpha} E\left[u_{1}^{2}(t)+2 u_{0}(t) u_{2}(t)\right] \\
& =-B v^{\alpha} E\left[\frac{k B^{2} t^{3 \alpha}}{\Gamma(3 \alpha+1)}+\frac{k^{2} A B \Gamma(2 \alpha+1) t^{4 \alpha}}{\Gamma(4 \alpha+1) \Gamma^{2}(\alpha+1)}\right. \\
& \left.+\frac{2 k^{2} A B \Gamma(3 \alpha+1) t^{4 \alpha}}{\Gamma(\alpha+1) \Gamma(2 \alpha+1) \Gamma(4 \alpha+1)}+\frac{2 k^{3} A^{2} \Gamma(2 \alpha+1) \Gamma(4 \alpha+1) t^{5 \alpha}}{\Gamma(3 \alpha+1) \Gamma(5 \alpha+1) \Gamma^{3}(\alpha+1)}\right] \\
& -A v^{\alpha} E\left[\frac{k^{2} B^{2} t^{4 \alpha}}{\Gamma^{2}(2 \alpha+1)}+\frac{2 k^{3} A B t^{5 \alpha}}{\Gamma(3 \alpha+1) \Gamma^{2}(\alpha+1)}+\frac{k^{4} A^{2} \Gamma^{2}(2 \alpha+1) t^{6 \alpha}}{\Gamma^{2}(3 \alpha+1) \Gamma^{4}(\alpha+1)}\right. \\
& +\frac{2 k t^{\alpha}}{\Gamma(\alpha+1)}\left(\frac{k B^{2} t^{3 \alpha}}{\Gamma(3 \alpha+1)}+\frac{k^{2} A B \Gamma(2 \alpha+1) t^{4 \alpha}}{\Gamma(4 \alpha+1) \Gamma^{2}(\alpha+1)}\right. \\
& \left.\left.+\frac{2 k^{2} A B \Gamma(3 \alpha+1) t^{4 \alpha}}{\Gamma(\alpha+1) \Gamma(2 \alpha+1) \Gamma(4 \alpha+1)}+\frac{2 k^{3} A^{2} \Gamma(2 \alpha+1) \Gamma(4 \alpha+1) t^{5 \alpha}}{\Gamma(3 \alpha+1) \Gamma(5 \alpha+1) \Gamma^{3}(\alpha+1)}\right)\right] \\
& u_{3}(t)=-k B^{3} v^{4 \alpha}-\frac{k^{2} A B^{2} v^{5 \alpha} \Gamma(2 \alpha+1)}{\Gamma^{2}(\alpha+1)}-\frac{2 k^{2} A B^{2} v^{5 \alpha} \Gamma(3 \alpha+1)}{\Gamma(\alpha+1) \Gamma(2 \alpha+1)} \\
& -\frac{2 k^{3} A^{2} B v^{6 \alpha} \Gamma(2 \alpha+1) \Gamma(4 \alpha+1)}{\Gamma(3 \alpha+1) \Gamma^{3}(\alpha+1)}-\frac{k^{2} A B^{2} v^{5 \alpha} \Gamma(4 \alpha+1)}{\Gamma^{2}(2 \alpha+1)} \\
& -\frac{2 k^{3} A^{2} B v^{6 \alpha} \Gamma(5 \alpha+1)}{\Gamma(3 \alpha+1) \Gamma^{2}(\alpha+1)}-\frac{k^{4} A^{3} v^{7 \alpha} \Gamma(6 \alpha+1) \Gamma^{2}(2 \alpha+1)}{\Gamma^{2}(3 \alpha+1) \Gamma^{4}(\alpha+1)} \\
& -\frac{2 k^{2} A B^{2} v^{5 \alpha} \Gamma(4 \alpha+1)}{\Gamma(\alpha+1) \Gamma(3 \alpha+1)}-\frac{2 k^{3} A^{2} B v^{6 \alpha} \Gamma(2 \alpha+1) \Gamma(5 \alpha+1)}{\Gamma(4 \alpha+1) \Gamma^{3}(\alpha+1)} \\
& -\frac{4 k^{3} A^{2} B v^{6 \alpha} \Gamma(3 \alpha+1) \Gamma(5 \alpha+1)}{\Gamma(2 \alpha+1) \Gamma(4 \alpha+1) \Gamma^{2}(\alpha+1)} \\
& -\frac{4 k^{4} A^{3} v^{7 \alpha} \Gamma(2 \alpha+1) \Gamma(4 \alpha+1) \Gamma(6 \alpha+1)}{\Gamma(3 \alpha+1) \Gamma(5 \alpha+1) \Gamma^{4}(\alpha+1)}
\end{aligned}
$$




$$
\begin{aligned}
& =-\frac{k B^{3} t^{4 \alpha}}{\Gamma(4 \alpha+1)}-\frac{k^{2} A B^{2} \Gamma(2 \alpha+1) t^{5 \alpha}}{\Gamma(5 \alpha+1) \Gamma^{2}(\alpha+1)}-\frac{2 k^{2} A B^{2} \Gamma(3 \alpha+1) t^{5 \alpha}}{\Gamma(\alpha+1) \Gamma(2 \alpha+1) \Gamma(5 \alpha+1)} \\
& -\frac{2 k^{3} A^{2} B \Gamma(2 \alpha+1) \Gamma(4 \alpha+1) t^{6 \alpha}}{\Gamma(3 \alpha+1) \Gamma(6 \alpha+1) \Gamma^{3}(\alpha+1)}-\frac{k^{2} A B^{2} \Gamma(4 \alpha+1) t^{5 \alpha}}{\Gamma(5 \alpha+1) \Gamma^{2}(2 \alpha+1)} \\
& -\frac{2 k^{3} A^{2} B \Gamma(5 \alpha+1) t^{6 \alpha}}{\Gamma(3 \alpha+1) \Gamma(6 \alpha+1) \Gamma^{2}(\alpha+1)}-\frac{k^{4} A^{3} \Gamma(6 \alpha+1) \Gamma^{2}(2 \alpha+1) t^{7 \alpha}}{\Gamma(7 \alpha+1) \Gamma^{2}(3 \alpha+1) \Gamma^{4}(\alpha+1)} \\
& -\frac{2 k^{2} A B^{2} \Gamma(4 \alpha+1) t^{5 \alpha}}{\Gamma(\alpha+1) \Gamma(3 \alpha+1) \Gamma(5 \alpha+1)}-\frac{2 k^{3} A^{2} B \Gamma(2 \alpha+1) \Gamma(5 \alpha+1) t^{6 \alpha}}{\Gamma(4 \alpha+1) \Gamma(6 \alpha+1) \Gamma^{3}(\alpha+1)} \\
& -\frac{4 k^{3} A^{2} B \Gamma(3 \alpha+1) \Gamma(5 \alpha+1) t^{6 \alpha}}{\Gamma(2 \alpha+1) \Gamma(4 \alpha+1) \Gamma(6 \alpha+1) \Gamma^{2}(\alpha+1)} \\
& -\frac{4 k^{4} A^{3} \Gamma(2 \alpha+1) \Gamma(4 \alpha+1) \Gamma(6 \alpha+1) t^{7 \alpha}}{\Gamma(3 \alpha+1) \Gamma(5 \alpha+1) \Gamma(7 \alpha+1) \Gamma^{4}(\alpha+1)} .
\end{aligned}
$$

Dengan demikian diperoleh solusi pendekatan

$$
\begin{aligned}
u(t) & =u_{0}(t)+u_{1}(t)+u_{2}(t)+u_{3}(t) \\
& =\frac{k t^{\alpha}}{\Gamma(\alpha+1)}-\frac{k B t^{2 \alpha}}{\Gamma(2 \alpha+1)}-\frac{k^{2} A \Gamma(2 \alpha+1) t^{3 \alpha}}{\Gamma(3 \alpha+1) \Gamma^{2}(\alpha+1)}+\frac{k B^{2} t^{3 \alpha}}{\Gamma(3 \alpha+1)} \\
& +\frac{k^{2} A B \Gamma(2 \alpha+1) t^{4 \alpha}}{\Gamma(4 \alpha+1) \Gamma^{2}(\alpha+1)}+\frac{2 k^{2} A B \Gamma(3 \alpha+1) t^{4 \alpha}}{\Gamma(\alpha+1) \Gamma(2 \alpha+1) \Gamma(4 \alpha+1)} \\
& +\frac{2 k^{3} A^{2} \Gamma(2 \alpha+1) \Gamma(4 \alpha+1) t^{5 \alpha}}{\Gamma(3 \alpha+1) \Gamma(5 \alpha+1) \Gamma^{3}(\alpha+1)}-\frac{k B^{3} t^{4 \alpha}}{\Gamma(4 \alpha+1)}-\frac{k^{2} A B^{2} \Gamma(2 \alpha+1) t^{5 \alpha}}{\Gamma(5 \alpha+1) \Gamma^{2}(\alpha+1)} \\
& -\frac{2 k^{2} A B^{2} \Gamma(3 \alpha+1) t^{5 \alpha}}{\Gamma(\alpha+1) \Gamma(2 \alpha+1) \Gamma(5 \alpha+1)}-\frac{2 k^{3} A^{2} B \Gamma(2 \alpha+1) \Gamma(4 \alpha+1) t^{6 \alpha}}{\Gamma(3 \alpha+1) \Gamma(6 \alpha+1) \Gamma^{3}(\alpha+1)} \\
& -\frac{k^{2} A B^{2} \Gamma(4 \alpha+1) t^{5 \alpha}}{\Gamma(5 \alpha+1) \Gamma^{2}(2 \alpha+1)}-\frac{2 k^{3} A^{2} B \Gamma(5 \alpha+1) t^{6 \alpha}}{\Gamma(3 \alpha+1) \Gamma(6 \alpha+1) \Gamma^{2}(\alpha+1)} \\
& -\frac{k^{4} A^{3} \Gamma(6 \alpha+1) \Gamma^{2}(2 \alpha+1) t^{7 \alpha}}{\Gamma(7 \alpha+1) \Gamma^{2}(3 \alpha+1) \Gamma^{4}(\alpha+1)}-\frac{2 k^{2} A B^{2} \Gamma(4 \alpha+1) t^{5 \alpha}}{\Gamma(\alpha+1) \Gamma(3 \alpha+1) \Gamma(5 \alpha+1)} \\
& -\frac{2 k^{3} A^{2} B \Gamma(2 \alpha+1) \Gamma(5 \alpha+1) t^{6 \alpha}}{\Gamma(4 \alpha+1) \Gamma(6 \alpha+1) \Gamma^{3}(\alpha+1)}-\frac{4 k^{3} A^{2} B \Gamma(3 \alpha+1) \Gamma(5 \alpha+1) t^{6 \alpha}}{\Gamma(2 \alpha+1) \Gamma(4 \alpha+1) \Gamma(6 \alpha+1) \Gamma^{2}(\alpha+1)} \\
& -\frac{4 k^{4} A^{3} \Gamma(2 \alpha+1) \Gamma(4 \alpha+1) \Gamma(6 \alpha+1) t^{7 \alpha}}{\Gamma(3 \alpha+1) \Gamma(5 \alpha+1) \Gamma(7 \alpha+1) \Gamma^{4}(\alpha+1)} .
\end{aligned}
$$

Selanjutnya akan dicari solusi dari persamaan (11) dengan $A=1, B=0$, dan $k=1$, maka persamaan diferensial fraksional non-linear berorde $\alpha$ sebagai berikut

$$
D^{\alpha} u(t)+u^{2}(t)=1
$$

dengan kondisi awal $u(0)=0$, diperoleh solusi persamaan diferensial fraksional Riccati sebagai berikut

$$
\begin{aligned}
u(t) & =\frac{t^{\alpha}}{\Gamma(\alpha+1)}-\frac{\Gamma(2 \alpha+1) t^{3 \alpha}}{\Gamma(3 \alpha+1) \Gamma^{2}(\alpha+1)}+\frac{2 \Gamma(2 \alpha+1) \Gamma(4 \alpha+1) t^{5 \alpha}}{\Gamma(3 \alpha+1) \Gamma(5 \alpha+1) \Gamma^{3}(\alpha+1)} \\
& -\frac{\Gamma(6 \alpha+1) \Gamma^{2}(2 \alpha+1) t^{7 \alpha}}{\Gamma(7 \alpha+1) \Gamma^{2}(3 \alpha+1) \Gamma^{4}(\alpha+1)}-\frac{4 \Gamma(2 \alpha+1) \Gamma(4 \alpha+1) \Gamma(6 \alpha+1) t^{7 \alpha}}{\Gamma(3 \alpha+1) \Gamma(5 \alpha+1) \Gamma(7 \alpha+1) \Gamma^{4}(\alpha+1)}
\end{aligned}
$$

Selanjutnya diambil suatu barisan berorde $a_{n}$ untuk melihat kekonvergenan barisan fungsi solusi persamaan diferensial fraksional

$$
\left(\alpha_{n}\right)=\left(\frac{n}{n+1}\right)
$$


maka persamaan diferensial fraksional berorde $a_{n}$ menjadi

$$
D^{\left(\frac{n}{n+1}\right)} u(t)+u^{2}(t)=1, n \in \mathbb{N}
$$

sehingga diperoleh $u_{n}(t)$ yaitu barisan fungsi solusi persamaan diferensial fraksional berorde $a_{n}$ sebagai berikut

$$
\begin{aligned}
u_{n}(t) & =\frac{t^{\left(\frac{n}{n+1}\right)}}{\Gamma\left(\left(\frac{n}{n+1}\right)+1\right)}-\frac{\Gamma\left(2\left(\frac{n}{n+1}\right)+1\right) t^{3\left(\frac{n}{n+1}\right)}}{\Gamma\left(3\left(\frac{n}{n+1}\right)+1\right) \Gamma^{2}\left(\left(\frac{n}{n+1}\right)+1\right)} \\
& +\frac{2 \Gamma\left(2\left(\frac{n}{n+1}\right)+1\right) \Gamma\left(4\left(\frac{n}{n+1}\right)+1\right) t^{5\left(\frac{n}{n+1}\right)}}{\Gamma\left(3\left(\frac{n}{n+1}\right)+1\right) \Gamma\left(5\left(\frac{n}{n+1}\right)+1\right) \Gamma^{3}\left(\left(\frac{n}{n+1}\right)+1\right)} \\
& -\frac{\Gamma\left(6\left(\frac{n}{n+1}\right)+1\right) \Gamma^{2}\left(2\left(\frac{n}{n+1}\right)+1\right) t^{7\left(\frac{n}{n+1}\right)}}{\Gamma\left(7\left(\frac{n}{n+1}\right)+1\right) \Gamma^{2}\left(3\left(\frac{n}{n+1}\right)+1\right) \Gamma^{4}\left(\left(\frac{n}{n+1}\right)+1\right)} \\
& -\frac{4 \Gamma\left(2\left(\frac{n}{n+1}\right)+1\right) \Gamma\left(4\left(\frac{n}{n+1}\right)+1\right) \Gamma\left(6\left(\frac{n}{n+1}\right)+1\right) t^{7\left(\frac{n}{n+1}\right)}}{\Gamma\left(3\left(\frac{n}{n+1}\right)+1\right) \Gamma\left(5\left(\frac{n}{n+1}\right)+1\right) \Gamma\left(7\left(\frac{n}{n+1}\right)+1\right) \Gamma^{4}\left(\left(\frac{n}{n+1}\right)+1\right)} .
\end{aligned}
$$

Selanjutnya ditunjukkan barisan fungsi $\left(u_{n}(t)\right)$ konvergen ke fungsi $u(t)$, dimana $u(t)$ adalah solusi persamaan diferensial fraksional Riccati orde $\alpha=1$.

$$
\begin{aligned}
u(t)= & t-\frac{t^{3}}{3}+\frac{2 t^{5}}{15}-\frac{t^{7}}{63}-\frac{4 t^{7}}{105} \\
\lim _{n \rightarrow \infty} u_{n}(t)= & \lim _{n \rightarrow \infty}\left\{\frac{t^{\left(\frac{n}{n+1}\right)}}{\Gamma\left(\left(\frac{\mathrm{n}}{\mathrm{n}+1}\right)+1\right)}-\frac{\Gamma\left(2\left(\frac{\mathrm{n}}{\mathrm{n}+1}\right)+1\right) t^{3\left(\frac{n}{n+1}\right)}}{\Gamma\left(3\left(\frac{\mathrm{n}}{\mathrm{n}+1}\right)+1\right) \Gamma^{2}\left(\left(\frac{\mathrm{n}}{\mathrm{n}+1}\right)+1\right)}\right. \\
& +\frac{2 \Gamma\left(2\left(\frac{n}{n+1}\right)+1\right) \Gamma\left(4\left(\frac{n}{n+1}\right)+1\right) t^{5\left(\frac{n}{n+1}\right)}}{\Gamma\left(3\left(\frac{n}{n+1}\right)+1\right) \Gamma\left(5\left(\frac{n}{n+1}\right)+1\right) \Gamma^{3}\left(\left(\frac{n}{n+1}\right)+1\right)} \\
& -\frac{\Gamma\left(6\left(\frac{n}{n+1}\right)+1\right) \Gamma^{2}\left(2\left(\frac{n}{n+1}\right)+1\right) t^{7\left(\frac{n}{n+1}\right)}}{\Gamma\left(7\left(\frac{n}{n+1}\right)+1\right) \Gamma^{2}\left(3\left(\frac{n}{n+1}\right)+1\right) \Gamma^{4}\left(\left(\frac{n}{n+1}\right)+1\right)} \\
& \left.-\frac{\left.4 \Gamma\left(2\left(\frac{n}{n+1}\right)+1\right) \Gamma\left(4\left(\frac{n}{n+1}\right)+1\right) \Gamma\left(6\left(\frac{n}{n+1}\right)+1\right) t^{7\left(\frac{n}{n+1}\right.}\right)}{\Gamma\left(3\left(\frac{n}{n+1}\right)+1\right) \Gamma\left(5\left(\frac{n}{n+1}\right)+1\right) \Gamma\left(7\left(\frac{n}{n+1}\right)+1\right) \Gamma^{4}\left(\left(\frac{n}{n+1}\right)+1\right)}\right\} \\
= & \frac{t}{\Gamma(1+1)}-\frac{\Gamma(2+1) t^{3}}{\Gamma(3+1) \Gamma^{2}(1+1)}+\frac{2 \Gamma(2+1) \Gamma(4+1) t^{5}}{\Gamma(3+1) \Gamma(5+1) \Gamma^{3}(1+1)} \\
& -\frac{\Gamma(6+1) \Gamma^{2}(2+1) t^{7}}{\Gamma(7+1) \Gamma^{2}(3+1) \Gamma^{4}(1+1)}-\frac{4 \Gamma(2+1) \Gamma(4+1) \Gamma(6+1) t^{7}}{\Gamma(3+1) \Gamma(5+1) \Gamma(7+1) \Gamma^{4}(1+1)} \\
= & \frac{t}{\Gamma(2)}-\frac{\Gamma(3) t^{3}}{\Gamma(4) \Gamma^{2}(2)}+\frac{2 \Gamma(3) \Gamma(5) t^{5}}{\Gamma(4) \Gamma(6) \Gamma^{3}(2)}-\frac{\Gamma(7) \Gamma^{2}(3) t^{7}}{\Gamma(8) \Gamma^{2}(4) \Gamma^{4}(2)} \\
& -\frac{4 \Gamma(3) \Gamma(5) \Gamma(7) t^{7}}{\Gamma(4) \Gamma(6) \Gamma(8) \Gamma^{4}(2)} \\
= & t-\frac{t^{3}}{3}+\frac{2 t^{5}}{15}-\frac{t^{7}}{63}-\frac{4 t^{7}}{105} \\
= & u(t) .
\end{aligned}
$$


- Apabila diambil empat suku yaitu $u_{0}, u_{1}, u_{2}$, dan $u_{3}$ maka diperoleh pola grafik sebagai berikut

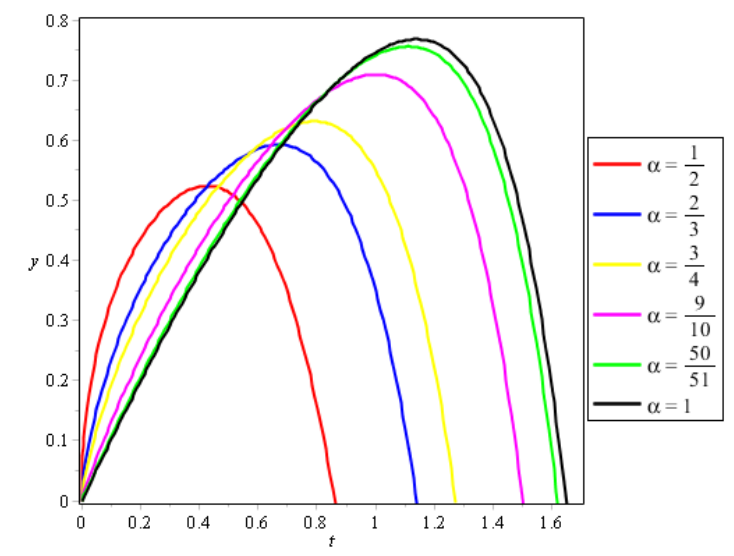

Gambar 1. Grafik fungsi solusi $D^{\frac{n}{n+1}} u(t)+u^{2}(t)=1$ untuk $u=u_{0}+u_{1}+u_{2}+u_{3}$.

- Apabila diambil tiga suku yaitu $u_{0}, u_{1}$, dan $u_{2}$ maka diperoleh pola grafik sebagai berikut

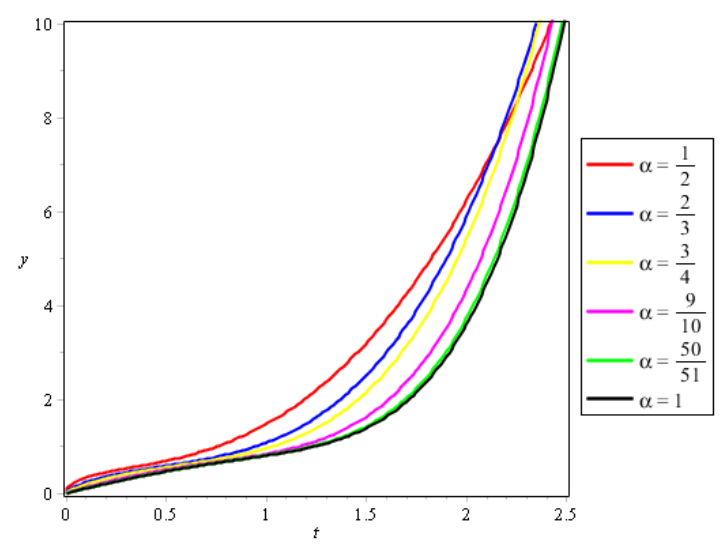

GAMBAR 2. Grafik fungsi solusi $D^{\frac{n}{n+1}} u(t)+u^{2}(t)=1$ untuk $u=u_{0}+u_{1}+u_{2}$.

- Apabila diambil dua suku yaitu $u_{0}$ dan $u_{1}$ maka diperoleh pola grafik sebagai berikut

- Apabila diambil satu suku yaitu $u_{0}$ maka diperoleh pola grafik sebagai berikut

Berdasarkan Gambar 1 sampai 4 dapat dilihat bahwa solusi persamaan diferensial fraksional Riccati dengan bentuk $D^{\left(\frac{n}{n+1}\right)} u(t)+u^{2}(t)=1$ menghasilkan pola grafik yang mendekati solusi persamaan diferensial fraksional berorde $\alpha=1$, sehingga barisan fungsi solusi persamaan diferensial fraksional Riccati dengan bentuk $D^{\left(\frac{n}{n+1}\right)} u(t)+u^{2}(t)=1$ konvergen ke fungsi solusi persamaan diferensial fraksional Riccati berdasarkan hasil analisis grafik dengan bentuk $D^{1} u(t)+u^{2}(t)=1$.

Selanjutnya ditunjukkan pola grafik solusi persamaan diferensial fraksional Riccati berorde $\alpha$ menggunakan Elzaki Decomposition Method dan menggunakan solusi eksak.

Diberikan persamaan diferensial fraksional Riccati berorde $\alpha$ berikut

$$
D^{\alpha} u(t)+u^{2}(t)=1 .
$$




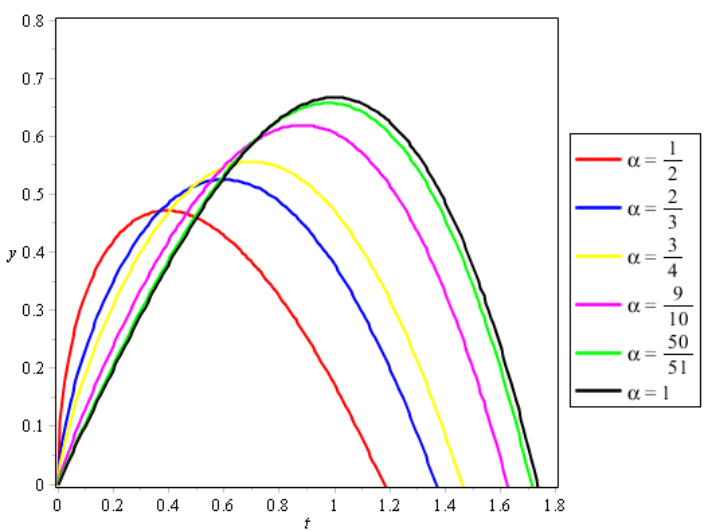

Gambar 3. Grafik fungsi solusi $D^{\frac{n}{n+1}} u(t)+u^{2}(t)=1$ untuk $u=u_{0}+u_{1}$.

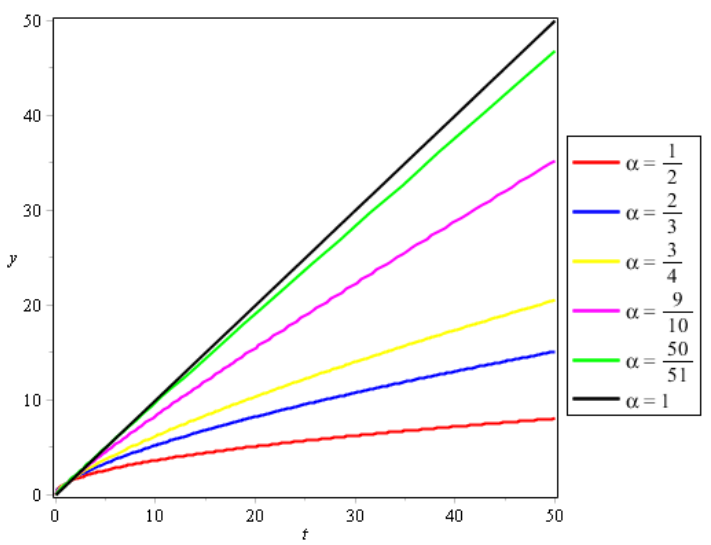

GAmbar 4. Grafik fungsi solusi $D^{\frac{n}{n+1}} u(t)+u^{2}(t)=1$ untuk $u=u_{0}$.

Bentuk umum solusi pendekatan dari persamaan diferensial fraksional Riccati tersebut yaitu

$$
\begin{aligned}
u(t) & =\frac{t^{\alpha}}{\Gamma(\alpha+1)}-\frac{\Gamma(2 \alpha+1) t^{3 \alpha}}{\Gamma(3 \alpha+1) \Gamma^{2}(\alpha+1)}+\frac{2 \Gamma(2 \alpha+1) \Gamma(4 \alpha+1) t^{5 \alpha}}{\Gamma(3 \alpha+1) \Gamma(5 \alpha+1) \Gamma^{3}(\alpha+1)} \\
& -\frac{\Gamma(6 \alpha+1) \Gamma^{2}(2 \alpha+1) t^{7 \alpha}}{\Gamma(7 \alpha+1) \Gamma^{2}(3 \alpha+1) \Gamma^{4}(\alpha+1)}-\frac{4 \Gamma(2 \alpha+1) \Gamma(4 \alpha+1) \Gamma(6 \alpha+1) t^{7 \alpha}}{\Gamma(3 \alpha+1) \Gamma(5 \alpha+1) \Gamma(7 \alpha+1) \Gamma^{4}(\alpha+1)} .
\end{aligned}
$$

Untuk $\alpha=1$ dengan menggunakan Elzaki Decomposition Method diperoleh solusi pendekatan sebagai berikut

$$
u_{E D M}(t)=t-\frac{t^{3}}{3}+\frac{2 t^{5}}{15}-\frac{t^{7}}{63}-\frac{4 t^{7}}{105}
$$

Untuk $\alpha=2$ dengan menggunakan Elzaki Decomposition Method diperoleh solusi pendekatan sebagai berikut

$$
u_{E D M}(t)=\frac{t^{2}}{2}-\frac{t^{6}}{120}+\frac{t^{10}}{10800}-\frac{t^{14}}{1123200}
$$

Untuk $\alpha=1$ diperoleh solusi eksak sebagai berikut

$$
u(t)=\frac{e^{2 t}-1}{e^{2 t}+1}
$$

Untuk $\alpha=2$ diperoleh solusi eksak sebagai berikut

$$
u(t)=\ln (\cosh (t))
$$




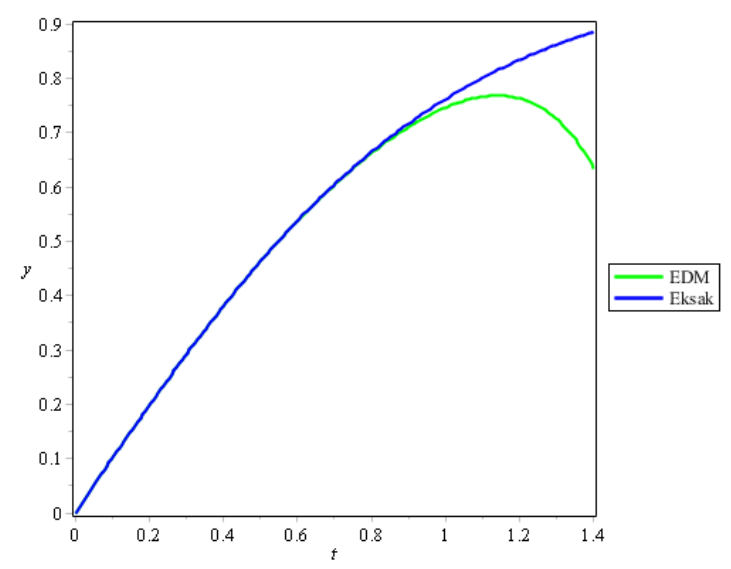

GAmBAR 5. Grafik solusi $D^{\alpha} u(t)+u^{2}(t)=1$ dengan $\alpha=1$.

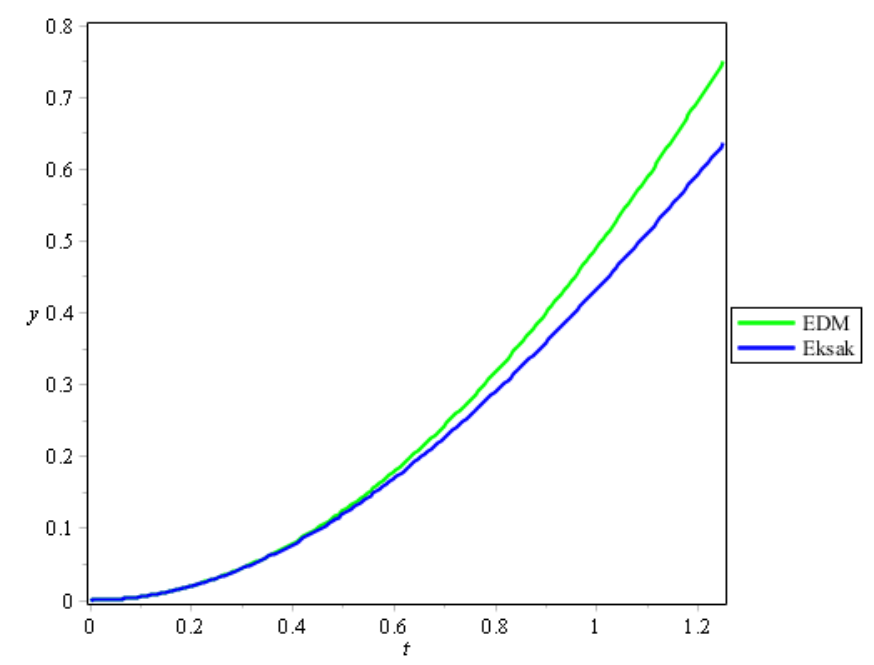

Gambar 6. Grafik solusi $D^{\alpha} u(t)+u^{2}(t)=1$ dengan $\alpha=2$.

Berdasarkan Gambar 5 dan 6, dapat dilihat grafik dari solusi pendekatan persamaan diferensial fraksional Riccati berbentuk $D^{\alpha} u(t)+u^{2}(t)=1$ dengan $\alpha=1$ dan $\alpha=2$ menggunakan Elzaki Decomposition Method (EDM) dan menggunakan solusi eksak.

\section{SIMPULAN}

Solusi dari persamaan diferensial fraksional Riccati berorde $\left(\alpha_{n}\right)$ dapat diperoleh dengan $0<\alpha \leq 2$ menggunakan Elzaki Decomposition Method (EDM). Jika barisan orde fraksional $\left(\alpha_{n}\right)$ konvergen ke $\alpha$ maka barisan fungsi solusi persamaan diferensial fraksional Riccati berorde $\left(\alpha_{n}\right)$ juga konvergen ke fungsi solusi persamaan diferensial fraksional Riccati berorde $\alpha$. Pola grafik dari barisan fungsi solusi persamaan diferensial fraksional Riccati berorde $\left(\alpha_{n}\right)$ bergerak menuju ke fungsi solusi persamaan diferensial fraksional Riccati berorde $\alpha$. Terdapat perbedaan dari beberapa pola grafik jika barisan solusi persamaan diferensial fraksional Riccati dibentuk dengan mengambil jumlah suku yang berbeda.

\section{Daftar Pustaka}

[1] Abdelkader Bouhassoun. Multistage telescoping decomposition method for solving fractional differential equations. International Journal of Applied Mathematics, 43(1), 2013. 
[2] Tarig M Elzaki and Mourad Chamekh. Solving nonlinear fractional differential equations using a new decomposition method. Univ J Appl Math Comput, 6:27-35, 2018.

[3] Muhamad Deni Johansyah, Julita Nahar, Asep K Supriatna, and Sudradjat Supian. Kajian dasar integral dan turunan fraksional riemann-liouville. In Prosiding Industrial Research Workshop and National Seminar, volume 8, pages 204-209, 2017.

[4] Muhamad Deni Johansyah, Herlina Napitupulu, Erwin Harahap, Ira Sumiati, and Asep K Supriatna. Solusi persamaan diferensial fraksional riccati menggunakan adomian decomposition method dan variational iteration method. Matematika, 18(1), 2019.

[5] Anatoliŭ Aleksandrovich Kilbas, Hari M Srivastava, and Juan J Trujillo. Theory and applications of fractional differential equations, volume 204. Elsevier, 2006.

[6] Kenneth S Miller and Bertram Ross. An introduction to the fractional calculus and fractional differential equations. Wiley, 1993.

[7] Shaher Momani and Nabil Shawagfeh. Decomposition method for solving fractional riccati differential equations. Applied Mathematics and Computation, 182(2):1083-1092, 2006.

[8] Zaid Odibat, Shaher Momani, and Vedat Suat Erturk. Generalized differential transform method: application to differential equations of fractional order. Applied Mathematics and Computation, 197(2):467-477, 2008.

[9] Zaid M Odibat and Shaher Momani. Application of variational iteration method to nonlinear differential equations of fractional order. International Journal of Nonlinear Sciences and Numerical Simulation, 7(1):27, 2006.

[10] NT Shawagfeh. The decomposition method for fractional. Journal of Fractional Calculus (ISSN 0918-5402), 16:27-33, 1999.

[11] Donald R Sherbert. Introduction to Real Analysis. Illinois: John Wiley \& Sons, Inc., 2011. 
